\title{
Correspondence
}

\section{Human retroviruses and paediatric disease}

Sir,

Thomson and Dalgleish ${ }^{1}$ discuss the management of human immunodeficiency virus (HIV) positive children with regard to immunisation and state that 'HIV positive children should never be given live viral vaccines'. In a review of the medical records of 213 children with symptoms of acquired immune deficiency syndrome (AIDS) or AIDS related complex (ARC), 171 had been immunised with live oral polio vaccine and 63 with measles, mumps, and rubella vaccine with no adverse effects. At least 38 of them received live vaccines after the onset of symptoms related to HIV. ${ }^{2}$ In addition, of the 71 children followed up in the European collaborative prospective study of infants born to mothers positive for HIV, 56 had received diphtheria/tetanus or diphtheria/tetanus/ polio vaccine (DTP), 30 Salk, 14 Sabin, eight hepatitis B, and one mumps vaccine. Of the five children who had developed AIDS or ARC, two had received DTP, two Salk, and one Sabin vaccine. No adverse reactions were reported in any child. ${ }^{3}$

The potential benefit of protecting these children from natural disease before their immune system is compromised led the World Health Organization and the Immune Practices Advisory Committee of the US Public Health Service to recommend that DTP, oral live polio, and measles vaccines should be given to asymptomatic children infected with HIV. ${ }^{2}{ }^{4}$ Live vaccines (oral polio, measles, and BCG) should only be withheld from children with symptoms of AIDS or ARC because of theoretical concerns about the competence of their immune system. Children, however, who live in a household with patients with AIDS or ARC should be given inactivated polio vaccine instead of oral polio vaccine because of the risk of secondary transmission. With regard to immunisation of infants positive for HIV, it is important therefore to distinguish between those who have symptoms related to HIV infection and those who do not.

All available information must be used before reaching decisions which will have far reaching implications, and we urge that caution be exercised before statements are made which may influence policy.

\section{References}

1 Thomson BJ, Dalgleish AG. Human retroviruses and paediatric disease. Arch Dis Child 1987;62:631-4.

2 Immunization Practices Advisory Committec. Immunization of children infected with Human T-lymphotropic virus type III/ lymphadenopathy associated virus. Pediatr Infect Dis 1987;6: 209-12.

${ }^{3}$ Mok JQ, Giaquinto C, De Rossi A, Grosch-Wörner I, Ades AE, Peckham CS. Infants born to HIV seropositive mothers - preliminary findings from a multi-centre European study. Lancet 1987;i:1164-8.
${ }^{4}$ Expanded Programme on Immunization. Joint WHO/UNICEF statement on immunization and AIDS. Weekly Epidemiological Record 1987;62:53-4.

Y D Senturia and C S Peckham Paediatric Epidemiology Department, Institute of Child Health, 30 Guilford Street, London WC1N 1EH

Sir,

Thomson and Dalgleish ${ }^{1}$ give advice on the immunisation of HIV positive children that is at variance with current practice and likely future official recommendations. They do not distinguish between children who are HIV positive but asymptomatic, and children who have developed clinical AIDS with associated immunodeficiency. In advising that 'HIV positive children should never be given live viral vaccines or BCG' they omit any reference to pertussis vaccine, which as a killed vaccine can be used with safety. Furthermore, recent experience suggests that:

(1) Even symptomatic HIV positive children may be given live vaccines with the exception of $\mathrm{BCG}$, as the benefits of protection appear to outweigh any risks from the vaccines. Inactivated vaccines should also be given in accordance with existing recommendations.

(2) Asymptomatic HIV antibody positive subjects may be given live vaccines for appropriate indications with the exception of BCG. If their parents or other household members are known to be immunocompromised (from AIDS or any other reason) the children should not receive oral polio vaccine because vaccine viruses are excreted by the recipient of the vaccine. These children should receive inactivated polio vaccine for routine immunisation.

(3) Children and young adults with AIDS or other clinical signs of HIV infection may be at risk of serious complications of infectious disease such as measles or varicella. If they are exposed to these diseases they should be given passive immunisation with appropriate immunoglobulin.

\section{Reference}

1 Thomson BJ, Dalgleish AG. Human retroviruses and paediatric disease. Arch Dis Child 1987;62:631-4.

A G M CAMPBELL Department of Child Health, University of Aberdeen, Aberdeen AB9 2ZD

Drs Thomson and Dalgleish comment:

We would like to thank Dr Senturia, Professor Peckham, and Dr Campbell for drawing attention to the controversial advice pertaining to live vaccines and HIV infected children in our annotation. The advice we quoted for the 